\title{
Increased telocytes involved in the proliferation of vascular smooth muscle cells in rat carotid artery balloon injury
}

\author{
Yanyan Li, Xiuxiu Zhang, Juan Gao, Han Xiao \& Ming Xu* \\ Department of Cardiology, Institute of Vascular Medicine, Peking University Third Hospital, Key Laboratory of Molecular Cardiovascular \\ Sciences, Ministry of Education; Key Laboratory of Cardiovascular Molecular Biology and Regulatory Peptides; Ministry of Health and \\ Beijing Key Laboratory of cardiovascular Receptors Research, Beijing 100191, China
}

Received April 1, 2016; accepted April 27, 2016

\begin{abstract}
Telocytes (TCs) are a novel type of interstitial cells that are thought to be involved in tissue regeneration and repair. However, the possible roles of TCs in vascular diseases remain unclear. In this study, we used a rat model of carotid artery balloon injury (CABI) to study the changes and potential roles of vascular TCs after vascular injury. Transmission electron microscopy (TEM) and CD34/vimentin immunolabeling were used to identify and quantify TCs in normal and injured carotid arteries. Quantitative immunofluorescence analysis revealed that, compared with the sham group, the number of TCs in the CABI group increased from $7.2 \pm 1.0$ to an average of $20.4 \pm 1.8$ per $1-\mathrm{mm}^{2}$ vascular area. The expression level of miR-24 in TCs was three times higher than in vascular smooth muscle cells (VSMCs). The percentage of VSMCs in S phase and G2/M phase increased by approximately $5 \%$ when VSMCs were incubated with the supernatant of TCs. The antagomir of miR-24 in TCs reduced the ratio of VSMCs in S phase and G2/M phase. This study illuminates the function of TCs in the proliferation of VSMCs.
\end{abstract}

blood vessel, telocyte, VSMCs, proliferation, vascular injury

Citation: Li, Y., Zhang, X., Gao, J., Xiao, H., and Xu, M. (2016). Increased telocytes involved in the proliferation of vascular smooth muscle cells in rat carotid artery balloon injury. Sci China Life Sci 59, 678-685. doi: 10.1007/s11427-016-5075-9

\section{INTRODUCTION}

VSMC proliferation is among the common cellular pathological bases of many vascular hyperplastic diseases, including hypertension, atherosclerosis and restenosis derived from vascular intervention. Particularly in vascular injury due to balloon angioplasty and endovascular stent implantation, the proliferation of VSMCs is an active part of the vascular repair process (Inoue and Node, 2009). Although VSMC phenotypic transformation and the stem cells/ progenitors of VSMCs have been considered to be the main factors in VSMC proliferation, the underlying mechanism of VSMC proliferation remains controversial. Recently, a new type of interstitial cells called TCs have attracted increasing attention because of their great potential in tissue

*Corresponding author (email: xuminghi@bjmu.edu.cn) regeneration and repair ( $\mathrm{Ja}$ et al., 2016; Li et al., 2014; Wang et al., 2014).

Increasing evidence has indicated that TCs are involved in the pathological processes of many diseases, including myocardial infarction (Manole et al., 2011; Zhao et al., 2013, 2014), heart failure (Richter and Kostin, 2015), renal ischemia-reperfusion injury ( $\mathrm{Li}$ et al., 2014), liver fibrosis (Fu et al., 2015), and others. However, the potential roles and mechanisms of TCs in the repair of vascular injury remained to be elucidated. In our previous study, TCs were observed to be located in the innermost layer of the tunica adventitia of the rat carotid artery, adjacent to the medial VSMCs. Thus, we hypothesized that the biological signal derived from TCs might be involved in VSMC proliferation.

In this study, we aimed to clarify the role of TCs in the repair of vascular injury and to explore the possible mecha- 
nisms. The rat model of CABI was established. TEM and immunostaining were used to identify vascular TCs in the model. The proliferation of VSMCs was analyzed by flow cytometry.

\section{RESULTS}

The number of TCs increased significantly in the CABI model

Vascular ultrasound and histomorphometric analysis showed that the luminal diameter of the injured CCA (common carotid artery) was significantly narrowed and neointima formed compared with the sham group (Figure $1 \mathrm{~A}$ and $\mathrm{B})$.

TEM showed that in the CCA, TCs were distributed in the connective tissue layer of the tunica adventitia, i.e., in the innermost layer of the adventitia and adjacent to the media. Compared with the sham group, the number of TCs showed a clear tendency toward increase in the CABI group
(Figure 1C).

The TCs of the CCA were identified by immunolabeling with CD34/vimentin. The numbers of TCs in the normal and injured CCA were compared using the immunoconfocal images, as shown in figure 1 (Figure 1D-I). As shown in Figure $1 \mathrm{~F}$ and I, CD34 and vimentin were co-localized at the junction of medial layer and adventitia in CCA. Immunostaining for CD34/vimentin and confocal microscopy found that the number of TCs in the CABI group was significantly higher than in the sham group. Quantitative analysis revealed that a $1 \mathrm{~mm}^{2}$ normal vessel contained 7.2 \pm 1.0 TCs. However, after CABI, the number of TCs rose dramatically and averaged $20.4 \pm 1.8$ per $1-\mathrm{mm}^{2}$ vascular area in the injured vessel (Figure 1J) $(P<0.001)$.

\section{Different immunophenotypes of vascular TCs, fibroblasts (FBs) and neurons}

In vitro, vascular TCs were identified by immunostaining with CD34/vimentin and CD34/PDGFR- $\beta$. TCs, FBs and
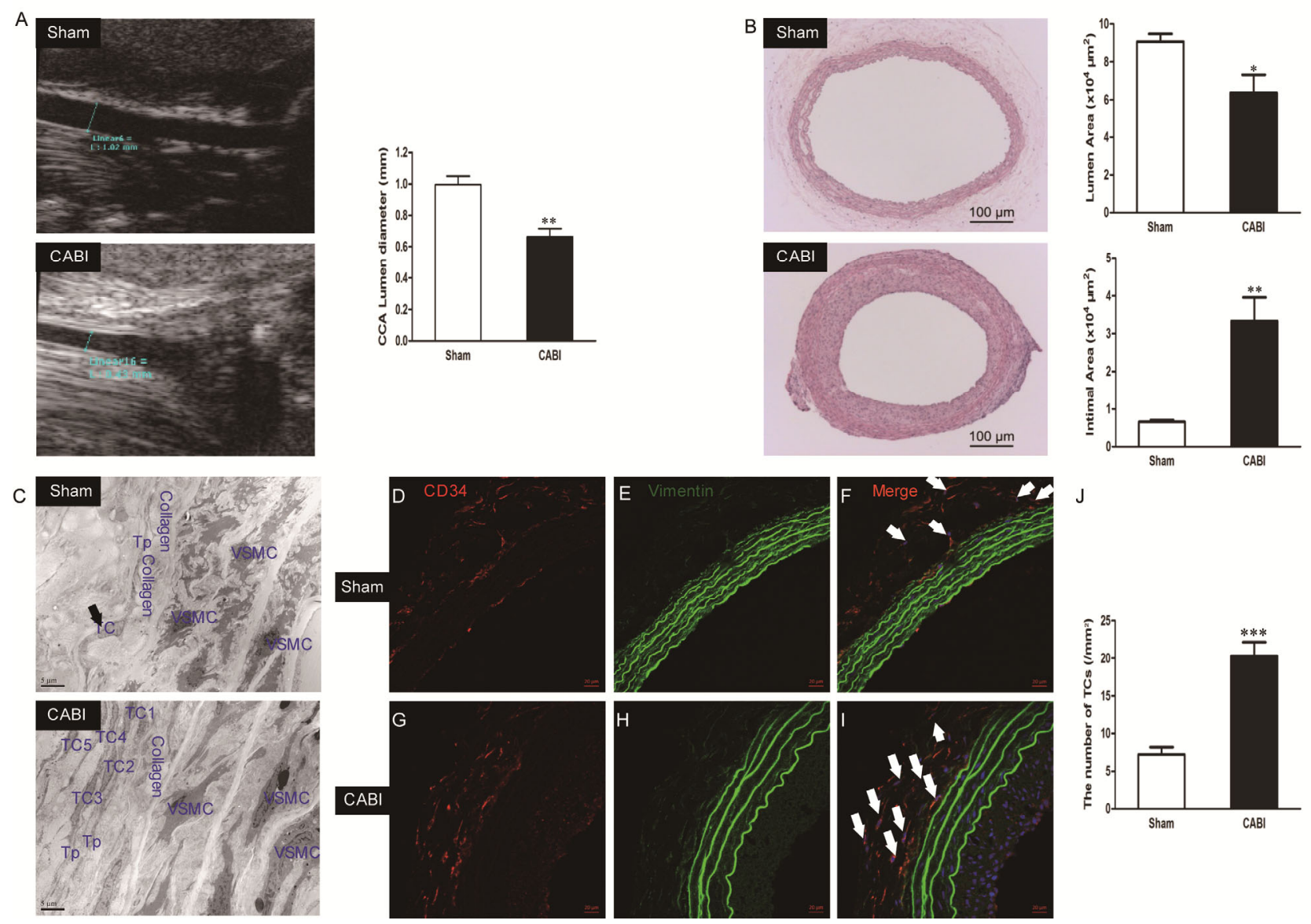

Figure 1 TCs were significantly increased in the CABI model. A, Vascular ultrasound performed 14 days after CABI and statistical histogram of lumen diameter of CCA based on vascular echo. B, Representative images of CCA sections stained with H\&E. Histomorphometric analysis showed lumen stenosis and neointimal formation in injured arteries. C, TEM image of the distribution of TCs in the CCA. Immunofluorescent confocal images showed that the CD34 (D and G) and vimentin $(\mathrm{E}$ and $\mathrm{H})$ double positive cells were significantly increased in the CABI group (I) than the sham group (F). White arrows denote TCs. Nuclei were stained blue with hoechst33342. Scale bar=20 $\mu \mathrm{m}$. J, Quantification of TCs in sham and CABI groups. Data are mean \pm SEM ( $n=7-9$ rats per group). (*, $P<0.05 ; * *, P<0.01$; ***, $P<0.001$ ). CABI, carotid artery balloon injury; CCA, common carotid artery; Tp, telopode; TEM, transmission electron microscope. 
neurons were distinguished through different double immunofluorescent methods. As shown in Figure 2A-F, vascular TCs were positive for CD34/vimentin and CD34/ PDGFR- $\beta$, while FBs and neurons were negative for CD34 (Figure 2G, J, M and P). FBs were positive for vimentin (Figure $2 \mathrm{H}$ ) and PDGFR- $\beta$ (Figure $2 \mathrm{~K}$ ), while neurons were only positive for PDGFR- $\beta$ (Figure $2 \mathrm{Q}$ ) and weakly positive for vimentin (Figure $2 \mathrm{~N}$ ). These data demonstrated the different immunophenotypes of TCs, FBs and neurons in primary culture.

\section{The supernatant of TCs promoted VSMCs proliferation}

Cell cycle analysis showed that the proportion of VSMCs in $\mathrm{S}$ phase and G2/M phase clearly increased when VSMCs were incubated with the supernatant of vascular TCs, $(10.68+3.53) \%$ vs. $(13.51+5.46) \%$, ((TCs-) vs. (TCs+), $P<0.05$ ) (Figure 3).

\section{miR-24 involved in TCs mediated the proliferation of VSMCs}

As shown in Figure 4A, the expression level of miR-24 in TCs was higher than in VSMCs based on the in situ hybridization of cells. Furthermore, when TCs were pre-treated with antagomir-24, the percentage of VSMCs in S phase and $\mathrm{G} 2 / \mathrm{M}$ phase clearly decreased $(12.76+4.92) \%$ vs. $(9.89+2.85) \%$, (antagomir-NC vs. antagomir-24, $P<0.05)$. Consistently, the ratio of VSMCs in G0/G1 phase increased considerably, $82.65 \%$ vs. $87.25 \%$, (antagomir-NC vs. an- tagomir-24, $P<0.05)$ (Figure 4B).

\section{DISCUSSION}

\section{Identification of the characteristics of TCs}

To explore the function of TCs, it is fundamental to identify their characteristics. TEM is considered to be an important means to identify TCs. However, fibroblasts, neurons and TCs all have a similar appearance, which cannot be distinguished by TEM. Therefore, combination with the immunophenotypic features of different cells was essential to differentiate TCs, fibroblasts and neurons. Double staining for immunofluorescence plays a key role in the identification of TCs, especially double positive immunostaining with CD34/vimentin or CD34/PDGFR- $\alpha(\beta)$ (Cretoiu and Popescu, 2014). In this study, vascular TCs were positive for CD34/vimentin and CD34/PDGFR- $\beta$. Fibroblasts were negative for CD34 but positive for vimentin and PDGFR- $\beta$. TCs and fibroblasts were different in functionality. Fibroblasts were mainly involved in the synthesis of collagen and the production of some matrix components, while TCs tended to act as active regulators in intercellular communication by homocellular/heterocellular junctions or shed vesicles. The function of TCs could be related to their various immunophenotypes. TCs were named interstitial neurons more than 100 years ago (Popescu and FaussonePellegrini, 2010). However, this study demonstrated that
TCs
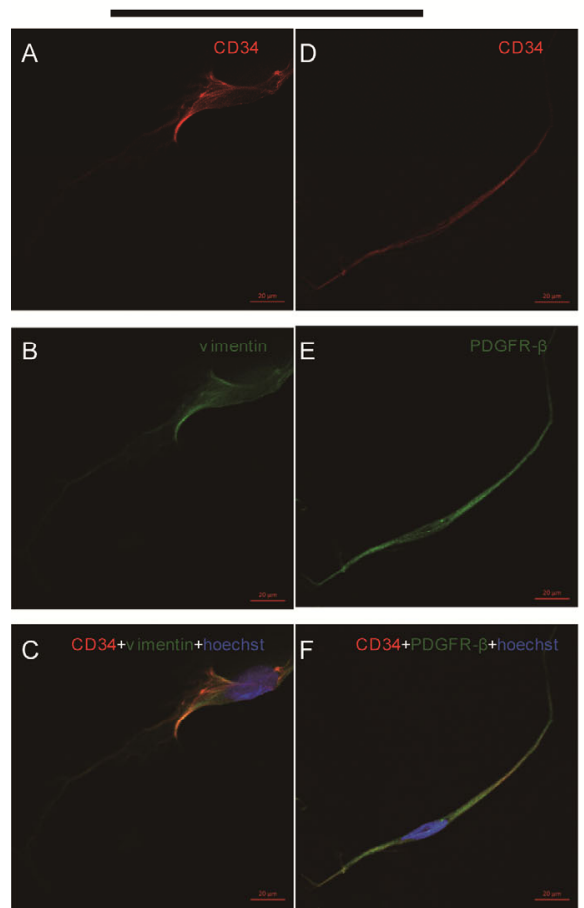

FBs
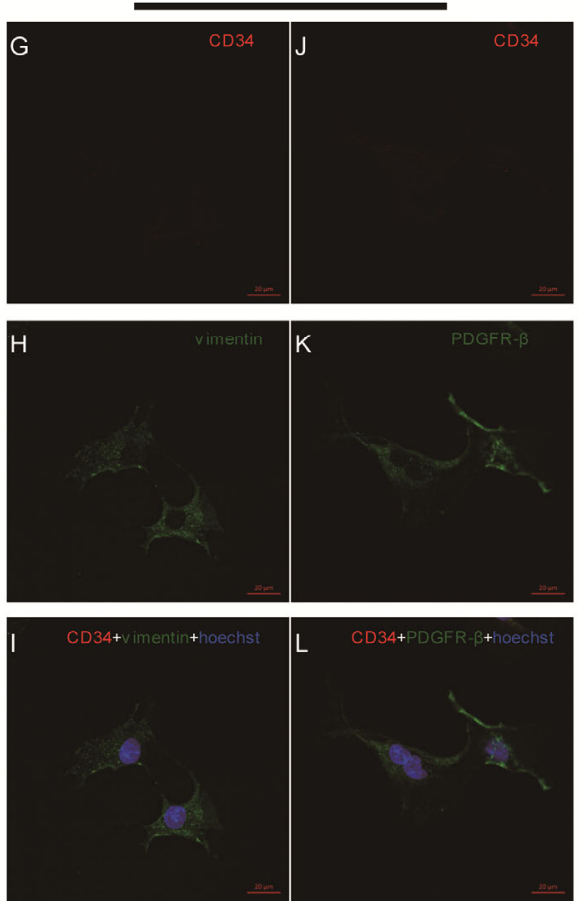

Neurons
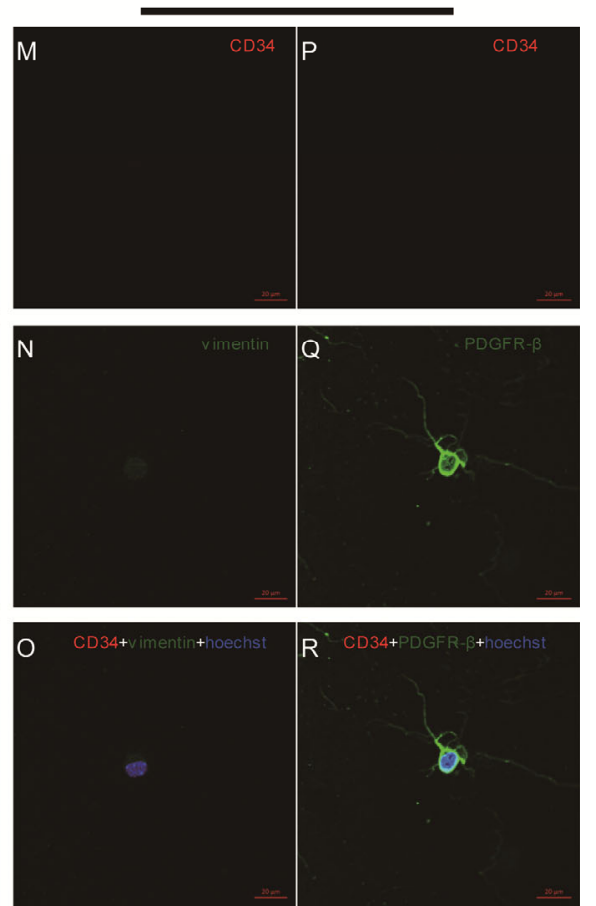

Figure 2 Different immunophenotypes of vascular TCs, fibroblasts (FBs) and cortical neurons. Confocal images show that TCs were positive for CD34 (red) (A and D), vimentin (B) and PDGFR- $\beta$ (green) (E). Co-localization of CD34/vimentin (C) and CD34/PDGFR- $\beta$ (F) in vascular TCs. FBs and neurons were negative for CD34 (red) (G and J, M and P). FBs were positive for vimentin (green) (H) and PDGFR- $\beta$ (green) (K). Neurons were weakly positive for vimentin (green) (N) and positive for PDGFR- $\beta$ (green) (Q). Nuclei were counterstained with hoechst33342 (blue). Scale bar=20 $\mu \mathrm{m}$. 


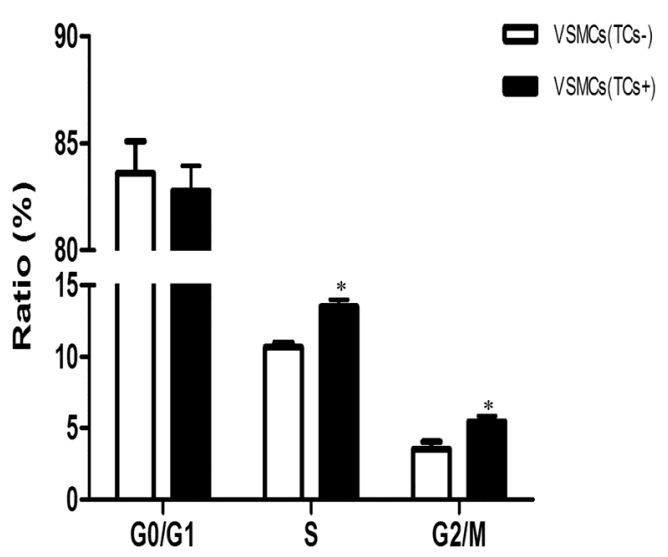

Figure 3 The supernatant of TCs promoted VSMC proliferation. VSMCs were incubated with or without the supernatant of vascular TCs for $48 \mathrm{~h}$, and the cell cycle of the VSMCs was analyzed. Data are mean $\pm \operatorname{SEM}(n=3)$ $(*, P<0.05)$. VSMCs, vascular smooth muscle cells.

neurons were negative for CD34 and weakly positive for vimentin, very different characteristics from TCs. Thus, shape combined with immunophenotypic characteristics plays a crucial role in distinguishing TCs from fibroblasts and neurons. In addition, vascular TCs were confirmed to express certain stem cell markers, including c-kit, nanog and sca-1 (Figure S1 in Supporting Information). This finding suggested that vascular TCs might have certain characteristics of stem cells. Studies have reported TCs to occupy a strategic position in relation to stem cell niches (Gherghiceanu et al., 2010) and to be able to guide and nurse stem cells (Gherghiceanu and Popescu, 2010; Zhou et al., 2014). Thus, TCs might be closely related to tissue regeneration and repair.

\section{TCs participate in the repair of vascular injury}

The repair of vascular injury is a process of neointimal formation and proliferation involving a cascade of cellular re- actions, and VSMC proliferation is highly important in this process. There are two main factors in VSMC proliferation after vascular injury, namely VSMC phenotypic switching and stem cells/progenitors of VSMCs. VSMCs display remarkable plasticity and undergo profound changes in phenotype during the repair of vascular injury, exhibiting a transition from the contractile phenotype to the synthetic phenotype (Alexander and Owens, 2012). The synthetic phenotype of VSMCs shows great proliferation and secretion ability. However, a lack of direct evidence has been available to trace the proliferative VSMCs from the synthetic phenotype of VSMCs. There was considerable controversy about the stem cells/progenitors of VSMCs, and the exact source, role and properties of these cells remained unclear. More and more studies reported that components of the adventitia participated in a wide range of vascular diseases (Majesky, 2015; Sano et al., 2016; Wang et al., 2016). In this study, TCs were observed to be located in the tunica adventitia. The number of TCs clearly increased in the injured carotid artery. Additionally, the supernatant of vascular TCs could promote the proliferation of VSMCs. Therefore, in addition to the phenotypic transformation of VSMCs and the stem cells/progenitors of VSMCs, vascular TCs were shown to be involved in the repair of vascular injury. However, the question remained: what is the mechanism by which TCs promote the proliferation of VSMCs?

\section{The underlying mechanisms by which TCs promote the proliferation of VSMCs}

The function of TCs is closely associated with microRNAs and secreting cytokines. Cardiac TCs contain some angiogenic miRs and promote angiogenesis after myocardial infarction (Manole et al., 2011). In another study, the microRNA signature in cardiac TCs was defined, and miR-193 was considered for differentiating TCs from other stromal cells (Cismasiu et al., 2011). Based on previous literature
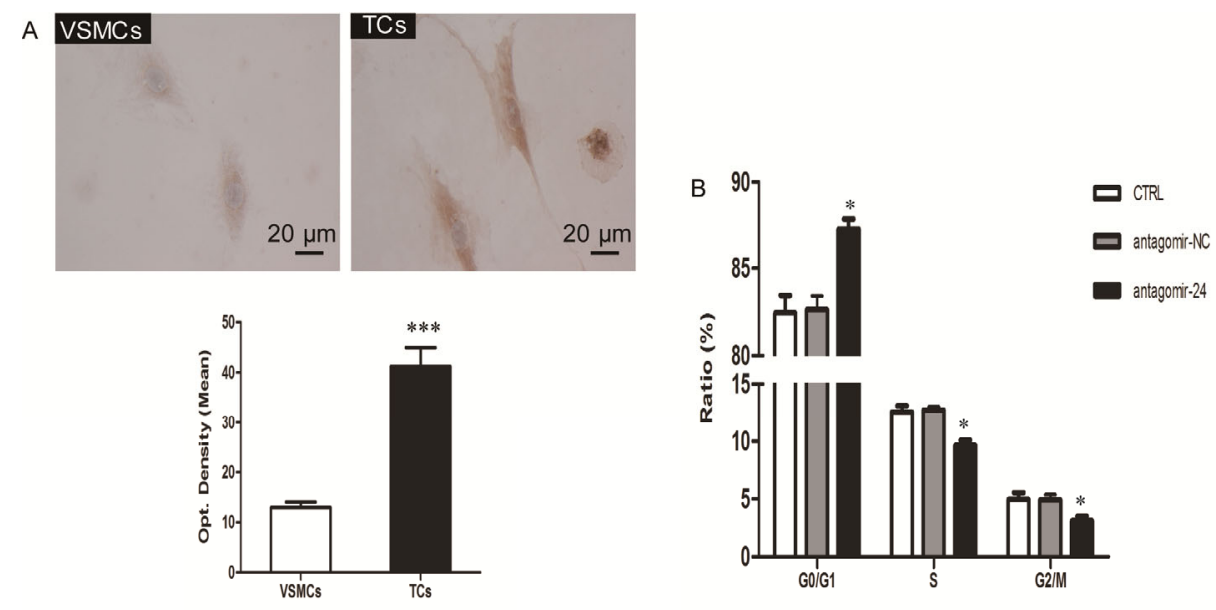

Figure 4 miR-24 is involved in the proliferation of VSMCs mediated by TCs. A, The expression of miR-24 in vascular TCs and VSMCs was quantified by in situ hybridization $(n=100)$. (***, $P<0.001)$. Scale bar $=20 \mu \mathrm{m}$. B, Cell cycle analysis of VSMCs with the supernatant of vascular TCs containing antagomir-NC or antagomir-24. Data are mean \pm SEM $(n=3)(*, P<0.05)$. VSMCs, vascular smooth muscle cells. 
reports, microRNAs participate in regulating the proliferation of VSMCs: for example, miR-24 (Chan et al., 2010), miR-221 (Liu et al., 2009), miR-146a (Sun et al., 2011), among others, can motivate the proliferation of VSMCs. Our results indicated that vascular TCs and VSMCs expressed miR-24, but the expression level of miR-24 in TCs was higher than in VSMCs. Interestingly, the supernatant of vascular TCs could not affect the proliferation of VSMCs when the TCs were pre-treated with antagomir-24. This result suggested that miR-24 was a key regulator for TC-mediated VSMCs proliferation. Whether the other microRNAs involved in the proliferation of VSMCs are mediated by TCs requires further study.

Cytokines are not only the key mediators for the roles of TCs but also are important factors in promoting the proliferation of VSMCs. Other studies have reported that TCs can secrete certain cytokines, including VEGF (vascular endothelial growth factor), IL-6 (interleukin-6), MIP-1 $\alpha$ (macrophage inflammatory protein $1-\alpha$ ), thus playing a modulatory role in stem cell proliferation and differentiation (Albulescu et al., 2015) or promoting the proliferation and angiogenesis of human pulmonary microvascular endothelial cells (Zheng et al., 2014). This study showed that the ratio of VSMCs in $\mathrm{S}$ and G2/M phase increased when VSMCs were incubated with the supernatant of TCs. Likewise, vascular TCs expressed VEGF (Figure S2 in Supporting Information). As a star molecule, VEGF plays a fundamental role in promoting angiogenesis, repairing injured vessels, and causing VSMC proliferation. Thus, vascular TCs might transmit some signals (including VEGF) to the other cells via direct contact or a paracrine mechanism, contributing to the proliferation of VSMCs.

In addition, the telopode, the very long and thin prolongation of TCs, might be involved in calcium uptake/release (Popescu and Faussone-Pellegrini, 2010), and intercellular calcium is an important regulator of VSMC proliferation (Rodriguez-Moyano et al., 2013). In our study, vascular TCs and VSMCs were co-cultured, and intercellular calcium was detected by Fluo 4-AM and Fura 2-AM. The results showed that $\mathrm{Ca}^{2+}$ concentration was not significantly different between the TCs and VSMCs, indicating that the $\mathrm{Ca}^{2+}$ of vascular TCs might not be a major factor in VSMC proliferation (Figure S3 in Supporting Information).

TCs are known to mediate intercellular communication through secreting cytokines or releasing extracellular vesicles. Recent evidence has been found that TCs can transfer extracellular vesicles loaded with microRNAs to stem cells from the heart and blood, and this epigenetic pathway is bidirectional (Cismasiu and Popescu, 2015). In this study, some extracellular vesicles of TCs were observed by living cell imaging and TEM (Figure S4 in Supporting Information). Based on previous studies, these vesicles might function as intercellular shuttles for biological signals (Cismasiu and Popescu, 2015; Manole et al., 2011). However, whether the vesicles of vascular TCs are loaded with
miR-24 or other molecules requires more study.

In conclusion, the number of TCs increased sharply in the CABI of rats. Using double immunofluorescent staining, our study demonstrated that vascular TCs were positive for CD34/vimentin and CD34/PDGFR- $\beta$, unlike fibroblasts and neurons. The supernatant of vascular TCs promoted VSMC proliferation, and miR-24 was shown to be an important regulator (Figure 5). Vascular TCs expressed some stem cell markers and VEGF and secreted vesicles, which might be other factors promoting VSMC proliferation. Thus, TCs served as active regulators in the repair process of vascular injury. Our study provides a new insight into the proliferation of VSMCs, and TCs might be a novel target for vascular hyperplastic diseases.

\section{MATERIALS AND METHODS}

\section{Animals}

Male Sprague Dawley (SD) rats (body weight, 250-300 g) and pregnant rats (E18d) were obtained from the laboratory animal department of Peking University Health Science Center.

\section{Rat carotid artery balloon injury (CABI) model}

The experimental protocol was modified from Tulis et al. (Tulis, 2007). Briefly, a rat was anesthetized, and the right CCA was exposed. For ligation of the distal end of the external carotid artery (ECA), the blood flow of the proximal CCA and internal carotid artery (ICA) was temporarily blocked with artery clamps. A'V'-shaped incision was made near the ligation of the ECA with an ophthalmic scissor. A

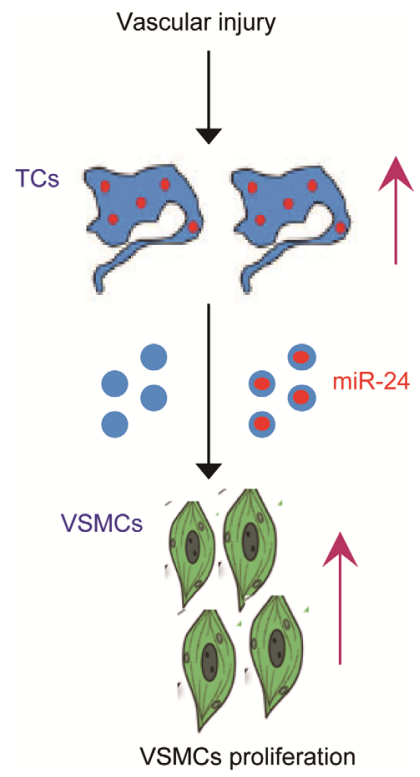

Figure 5 A diagram that TCs mediated the proliferation of VSMCs after vascular injury. The number of TCs increased significantly after vascular injury. The supernatant of TCs promoted VSMC proliferation, and miR-24 was shown to be an important regulator in this process. 
PTCA balloon catheter $(1.5 \mathrm{~mm} \times 15 \mathrm{~mm}$, Maverick, USA) was introduced via the arteriotomy incision, then slowly advanced to the proximal edge of the CCA. To produce carotid artery injury, the balloon was inflated with air and withdrawn three times, and ligation was performed on the ECA proximal to the bifurcation. The blood flow in the CCA and ICA was then restored. In the sham group, the CCA was isolated but not injured.

Fourteen days after the operation, the lumen diameter of the CCA was measured using a VisualSonics Vevo 770 cardiovascular ultrasound system (VisualSonics, Inc., Canada).

\section{Morphometric analysis for neointimal lesion formation}

Small fragments (approximately $0.5-1 \mathrm{~cm}$ ) of the CCA were fixed with $10 \%$ neutral formalin for hematoxylin-eosin (H\&E) staining. The specimen was cut $5 \mu \mathrm{m}$ thick. Morphometric analysis using a computerized image analysis system (Image Pro Plus 6.0, Media Cybernetics, USA) was performed in segments, as described previously ( $\mathrm{Ji}$ et al., 2007). The lumen area and neointimal area were measured and calculated.

\section{Transmission electron microscopy (TEM)}

An anesthetized rat was fixed, and the thoracic cavity was opened. To fix the blood vessels in situ, $20 \mathrm{~mL}$ of physiological saline (containing heparin sodium 240 IU per $20 \mathrm{~mL}$ ) and $10 \mathrm{~mL} \mathrm{4 \%}$ paraformaldehyde solution was perfused through a puncture in the left ventricle. Then, the CCA was removed and cut into small fragments (approximately $1 \mathrm{~mm}^{3}$ ). The fragments were washed with ice phosphate buffer and fixed in $3 \%$ glutaraldehyde at $4^{\circ} \mathrm{C}$ overnight. The samples were postfixed with $1 \%$ osmium tetroxide for $2 \mathrm{~h}$ and dehydrated in a graded series of acetone, followed by saturation with a mixture of different proportions of acetone and epoxy resin, embedding with a board containing epoxy resin. The ultrathin sections $(70 \mathrm{~nm})$ were made using a LEICA EM UC7 (Germany) and stained with uranyl acetate and lead citrate for $30 \mathrm{~min}$. The sections were observed and photographed under TEM (JEM-1230, Japan).

\section{Isolation and primary culture of vascular TCs, VSMCs, fibroblasts and neurons}

The CCA was isolated under sterile conditions. After rinsing with fresh phosphate-buffered saline (PBS) 5-6 times, the vessel was cut along the longitudinal axis. The vascular intima and tunica media were scraped. The intima and adventitia were removed to isolate the VSMCs. The vessel was cut into 3-4 segments. The sections were then minced into $1 \mathrm{~mm}^{3}$ pieces and digested with $1 \mathrm{mg} \mathrm{mL}^{-1}$ collagenase type II (Gibco, USA) for 6-8 h on an orbital shaker at $37^{\circ} \mathrm{C}$. Dissociated cells were collected by centrifugation (168×g, $10 \mathrm{~min})$. Cells were resuspended in DMEM (Gibco) supplemented with $10 \%$ fetal bovine serum (FBS) (HyClone, USA), $100 \mathrm{U} \mathrm{mL}^{-1}$ penicillin, and $100 \mu \mathrm{g} \mathrm{mL}^{-1}$ streptomycin (Gibco), then cultured in a humidified atmosphere of $5 \% \mathrm{CO}_{2}$ at $37^{\circ} \mathrm{C}$ for $2 \mathrm{~h}$ to purify vascular TCs and allow fibroblast attachment. The suspension (containing TCs) was collected and plated. To further purify the vascular TCs, CD34 ${ }^{+} / \mathrm{c}-\mathrm{kit}^{+}$cells were sorted through flow cytometry (BD FACSAria ${ }^{\mathrm{M}}$ II). The morphology of the cells was observed using an inverted phase contrast microscope (LEICA DMIRE2, Germany) (Figure S5 in Supporting Information).

Cortical neurons were aseptically obtained from pregnant rats (E18d) and digested with $0.2 \%$ trypsin (Gibco) for 30 $\min$ at $37^{\circ} \mathrm{C}$, followed by trituration with a flame-polished Pasteur pipette. Dissociated cells were collected by centrifugation $(168 \times g, 5 \mathrm{~min})$. The neurons were resuspended in plating medium (DMEM containing 10\% FBS), then plated onto coverslips coated with poly-D-lysine (Sigma-Aldrich, USA). After $4 \mathrm{~h}$, the medium was replaced with Neurobasal medium supplemented with $2 \%$ B27, 2 mmol L ${ }^{-1}$ Gluta-

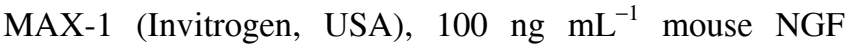
(Promega, USA), $100 \mathrm{U} \mathrm{mL}^{-1}$ penicillin and $100 \mu \mathrm{g} \mathrm{mL}$ streptomycin. After plating for 18-24 h, a final concentration of $10 \mu \mathrm{mol} \mathrm{L}{ }^{-1}$ Arac (Sigma-Aldrich) was added to the culture medium (Wang et al., 2015).

\section{Immunofluorescent staining for $\mathrm{CCA}$ and cells}

After fixing with $4 \%$ paraformaldehyde and dehydration with $20 \%$ sucrose solution, the tissue samples were embedded in O.C.T compound (SAKURA, USA) and frozen with liquid nitrogen, then cut at a thickness of $7 \mu \mathrm{m}$ through LEICA CM3050 S (Germany). The tissues were postfixed in acetone for $10 \mathrm{~min}$, and then $0.2 \%$ Triton X-100 (Sigma-Aldrich) was added for $15 \mathrm{~min}$. The sections were incubated with $1 \%$ bovine serum albumin (BSA) for $30 \mathrm{~min}$. After rinsing in PBS, the samples were incubated at $4^{\circ} \mathrm{C}$ overnight with primary antibodies against CD34 (Abcam, USA) and vimentin (FITC) (Abcam). Alexa Fluor ${ }^{\circledR}$ goat anti-rabbit IgG 568 (Invitrogen) served as the secondary antibody in the detection system. The nuclei were stained with hoechst33342 (Sigma-Aldrich). The negative controls were obtained using PBS instead of the primary antibodies. The samples were examined by confocal laser microscopy using LSM 780 (Zeiss, Germany).

Cells were grown on coverslips. After being washed with PBS three times, the cells were fixed with $4 \%$ paraformaldehyde for $15 \mathrm{~min}$. The subsequent steps were the same as above.

\section{Quantitative immunofluorescent microscopy for TCs in CCA}

We counted the positive co-expression of CD34 and vimentin of cells having a hoechst33342-positive nucleus and thin prolongations. Each group contained at least 7 samples. At least ten random fields in each section were analyzed. The number of TCs was calculated per $1-\mathrm{mm}^{2}$ vascular area. 


\section{In situ hybridization for TCs and VSMCs}

miR-24 expression was detected using a DIG labeling and detection kit (BOSTER, China). Standard procedures were performed according to the manufacturer's instructions. The expression level of miR-24 was calculated in 100 vascular TCs and VSMCs each.

\section{Transfection}

Vascular TCs were transfected when the cells were in good condition. Antagomir-24 (RIBOBIO, China) or antagomir-NC (RIBOBIO) at a final concentration of $100 \mathrm{nmol} \mathrm{L}^{-1}$ and Lipofectamine 2000 (Invitrogen) were incubated separately with Opti-MEM I (Invitrogen) for $5 \mathrm{~min}$, then mixed and incubated for $20 \mathrm{~min}$. The cell medium was replaced for the transfection reaction. The medium was refreshed after 4 h. The supernatant of the TCs was collected and centrifuged to remove debris after $48 \mathrm{~h}$. The supernatant was stored at $-80^{\circ} \mathrm{C}$.

\section{Cell cycle analysis}

VSMCs were cultured with DMEM not containing FBS when the cells grew to a confluency of $60 \%-70 \%$. After $12 \mathrm{~h}$, the VSMCs were treated with the supernatant of vascular TCs containing or not containing miR-24 for $48 \mathrm{~h}$. VSMCs were then collected and fixed with $70 \%$ ethanol at $4^{\circ} \mathrm{C}$ overnight. Cells were treated with $0.2 \mathrm{mg} \mathrm{mL}^{-1} \mathrm{RNase}$ $\mathrm{A}$ and incubated at $37^{\circ} \mathrm{C}$ for $30 \mathrm{~min}$, followed by the addition of 7-aminoactinomycin D (BD) and incubation for 10 min. The cell cycle of the VSMCs was detected using a BD FACSAria $^{\mathrm{M}}$ II.

\section{Statistical analysis}

Data are presented as the mean \pm SEM. For two-group comparison, the unpaired two-tailed Student's test was used. PRISM 5.0 (GRAPHPAD software, USA) was used for statistical analysis. $P<0.05$ was considered a statistically significant difference.

Compliance and ethics The author(s) declare that they have no conflicts of interest. All experimental protocols using animals were approved by the Institutional Animal Care and Use Committee of Peking University Health Science Center.

Acknowledgements Thanks to Prof. Wang and her colleagues (Electron Microscope Center of Peking University First Hospital, Beijing, China) for their help with electron microscopy techniques. This work was supported by the National Natural Science Foundation of China (91339105), the Beijing Natural Science Foundation (7142165), and the Science and Technology Commission of Beijing Municipality (z141100000214006).

Albulescu, R., Tanase, C., Codrici, E., Popescu, D.I., Cretoiu, S.M., and Popescu, L.M. (2015). The secretome of myocardial telocytes modulates the activity of cardiac stem cells. J Cell Mol Med 19, 1783-1794.

Alexander, M.R., and Owens, G.K. (2012). Epigenetic control of smooth muscle cell differentiation and phenotypic switching in vascular development and disease. Annu Rev Physiol 74, 13-40.
Chan, M.C., Hilyard, A.C., Wu, C., Davis, B.N., Hill, N.S., Lal, A., Lieberman, J., Lagna, G., and Hata, A. (2010). Molecular basis for antagonism between PDGF and the TGFbeta family of signalling pathways by control of miR-24 expression. EMBO J 29, 559-573.

Cismasiu, V.B., and Popescu, L.M. (2015). Telocytes transfer extracellular vesicles loaded with microRNAs to stem cells. J Cell Mol Med 19, $351-358$.

Cismasiu, V.B., Radu, E., and Popescu, L.M. (2011). miR-193 expression differentiates telocytes from other stromal cells. J Cell Mol Med 15, 1071-1074.

Cretoiu, S.M., and Popescu, L.M. (2014). Telocytes revisited. Biomol Concepts 5, 353-369.

Fu, S., Wang, F., Cao, Y., Huang, Q., Xiao, J., Yang, C., and Popescu, L.M. (2015). Telocytes in human liver fibrosis. J Cell Mol Med 19, 676-683.

Gherghiceanu, M., Manole, C.G., and Popescu, L.M. (2010). Telocytes in endocardium: electron microscope evidence. J Cell Mol Med 14, 2330-2334.

Gherghiceanu, M., and Popescu, L.M. (2010). Cardiomyocyte precursors and telocytes in epicardial stem cell niche: electron microscope images. J Cell Mol Med 14, 871-877.

Inoue, T., and Node, K. (2009). Molecular basis of restenosis and novel issues of drug-eluting stents. Circ J 73, 615-621.

Ja, K.M., Miao, Q., Zhen Tee, N.G., Lim, S.Y., Nandihalli, M., C, J.A.R., Mehta, A., and Shim, W. (2016). iPSC-derived human cardiac progenitor cells improve ventricular remodelling via angiogenesis and interstitial networking of infarcted myocardium. J Cell Mol Med 20, 323-332.

Ji, R., Cheng, Y., Yue, J., Yang, J., Liu, X., Chen, H., Dean, D.B., and Zhang, C. (2007). MicroRNA expression signature and antisense-mediated depletion reveal an essential role of microRNA in vascular neointimal lesion formation. Circ Res 100, 1579-1588.

Li, L., Lin, M., Wang, R., Zhang, C., Qi, G., Xu, M., Rong, R., and Zhu, T. (2014). Renal telocytes contribute to the repair of ischemically injured renal tubules. J Cell Mol Med 18, 1144-1156.

Liu, X., Cheng, Y., Zhang, S., Lin, Y., Yang, J., and Zhang, C. (2009). A necessary role of miR-221 and miR-222 in vascular smooth muscle cell proliferation and neointimal hyperplasia. Circ Res 104, 476-487.

Majesky, M.W. (2015). Adventitia and perivascular cells. Arterioscler Thromb Vasc Biol 35, e31-35.

Manole, C.G., Cismasiu, V., Gherghiceanu, M., and Popescu, L.M. (2011). Experimental acute myocardial infarction: telocytes involvement in neo-angiogenesis. J Cell Mol Med 15, 2284-2296.

Popescu, L.M., and Faussone-Pellegrini, M.S. (2010). TELOCYTES-a case of serendipity: the winding way from Interstitial Cells of Cajal (ICC), via Interstitial Cajal-Like Cells (ICLC) to TELOCYTES. J Cell Mol Med 14, 729-740.

Richter, M., and Kostin, S. (2015). The failing human heart is characterized by decreased numbers of telocytes as result of apoptosis and altered extracellular matrix composition. J Cell Mol Med 19, 2597-2606.

Rodriguez-Moyano, M., Diaz, I., Dionisio, N., Zhang, X., Avila-Medina, J., Calderon-Sanchez, E., Trebak, M., Rosado, J.A., Ordonez, A., and Smani, T. (2013). Urotensin-II promotes vascular smooth muscle cell proliferation through store-operated calcium entry and EGFR transactivation. Cardiovasc Res 100, 297-306.

Sano, M., Unno, N., Sasaki, T., Baba, S., Sugisawa, R., Tanaka, H., Inuzuka, K., Yamamoto, N., Sato, K., and Konno, H. (2016). Topologic distributions of vasa vasorum and lymphatic vasa vasorum in the aortic adventitia-Implications for the prevalence of aortic diseases. Atherosclerosis 247, 127-134.

Sun, S.G., Zheng, B., Han, M., Fang, X.M., Li, H.X., Miao, S.B., Su, M., Han, Y., Shi, H.J., and Wen, J.K. (2011). miR-146a and Kruppel-like factor 4 form a feedback loop to participate in vascular smooth muscle cell proliferation. EMBO Rep 12, 56-62.

Tulis, D.A. (2007). Rat carotid artery balloon injury model. Methods Mol Med 139, 1-30.

Wang, F., Song, Y., Bei, Y., Zhao, Y., Xiao, J., and Yang, C. (2014). Telocytes in liver regeneration: possible roles. J Cell Mol Med 18, 1720-1726.

Wang, G.Q., Cen, C., Li, C., Cao, S., Wang, N., Zhou, Z., Liu, X.M., Xu, 
Y., Tian, N.X., Zhang, Y., Wang, J., Wang, L.P., and Wang, Y. (2015). Deactivation of excitatory neurons in the prelimbic cortex via Cdk5 promotes pain sensation and anxiety. Nat Commun 6, 7660.

Wang, X., Chen, L., Liu, J., Yan, T., Wu, G., Xia, Y., Zong, G., and Li, F. (2016). In vivo treatment of rat arterial adventitia with interleukin 1 beta induces intimal proliferation via the JAK2/STAT3 signaling pathway. Mol Med Rep 13, 3451-3458.

Zhao, B., Chen, S., Liu, J., Yuan, Z., Qi, X., Qin, J., Zheng, X., Shen, X., Yu, Y., Qnin, T.J., Chan, J.Y., and Cai, D. (2013). Cardiac telocytes were decreased during myocardial infarction and their therapeutic effects for ischaemic heart in rat. J Cell Mol Med 17, 123-133.

Zhao, B., Liao, Z., Chen, S., Yuan, Z., Yilin, C., Lee, K.K., Qi, X., Shen,
X., Zheng, X., Quinn, T., and Cai, D. (2014). Intramyocardial transplantation of cardiac telocytes decreases myocardial infarction and improves post-infarcted cardiac function in rats. J Cell Mol Med 18, 780-789.

Zheng, Y., Chen, X., Qian, M., Zhang, M., Zhang, D., Bai, C., Wang, Q., and Wang, X. (2014). Human lung telocytes could promote the proliferation and angiogenesis of human pulmonary microvascular endothelial cells in vitro. Mol Cell Ther doi: 10.1186/2052-8426-2-3.

Zhou, J., Wang, Y., Zhu, P., Sun, H., Mou, Y., Duan, C., Yao, A., Lv, S., and Wang, C. (2014). Distribution and characteristics of telocytes as nurse cells in the architectural organization of engineered heart tissues. Sci China Life Sci 57, 241-247.

Open Access This article is distributed under the terms of the Creative Commons Attribution License which permits any use, distribution, and reproduction in any medium, provided the original author(s) and source are credited.

\section{SUPPORTING INFORMATION}

Figure S1 Vascular TCs expressed certain stem cell markers.

Figure S2 Vascular TCs expressed VEGF.

Figure S3 Intercellular $\mathrm{Ca}^{2+}$ in vascular TCs and VSMCs.

Figure S4 Vascular TCs could secrete vesicles.

Figure S5 The morphology of cultured vascular TCs under light microscope.

The supporting information is available online at life.scichina.com and link.springer.com. The supporting materials are published as submitted, without typesetting or editing. The responsibility for scientific accuracy and content remains entirely with the authors. 